\title{
Modelling and testing of a soft suspension design for a reaction/momentum wheel assembly
}

\author{
Wei-Yong Zhou ${ }^{\mathrm{a}, \mathrm{b}, *}$, Guglielmo S. Aglietti ${ }^{\mathrm{a}}$, Zhe Zhang ${ }^{\mathrm{a}}$ \\ a Astronautics Research Group, School of Engineering Science, University of Southampton, Southampton, Hampshire SO17 1BJ, UK \\ ${ }^{\mathrm{b}}$ College of Aerospace and Material Engineering, National University of Defense Technology, No. 47 Yanwachi Street, ChangSha 410073, \\ People's Republic of China
}

\section{A R T I C L E I N F O}

\section{Article history:}

Received 26 August 2010

Received in revised form

8 January 2011

Accepted 28 March 2011

Handling Editor: K. Worden

Available online 18 May 2011

\begin{abstract}
A B S T R A C T
The micro-vibrations generated by equipment onboard spacecraft can significantly affect the performance of sensitive payloads; in particular mid to high frequency band microvibrations, are difficult to model and control. This study focuses on the micro-vibrations emitted by a wheel assembly (WA) implementing a soft (flexible) suspension system. Both the soft and a "conventional" rigid design were tested using a Seismic Micro-Vibration Measurement System (SMVMS). A Newton-Euler method was employed to develop an analytical model of the WA and the test system, which was then used to model and analyze the micro-vibrations emitted by the reaction/momentum WA, due to the static and dynamic imbalance of the flywheel. When compared with the traditional "rigid" design, the soft suspension system is shown to effectively reduce the high frequency disturbances, and the mathematical model effectively represents the fundamental harmonic disturbances. In addition, the results confirm that the SMVMS shows relatively high measurement accuracy.
\end{abstract}

Crown Copyright @ 2011 Published by Elsevier Ltd. All rights reserved.

\section{Introduction}

The micro-vibrations generated by mechanical systems onboard spacecraft, for example reaction/momentum Wheel Assemblies (WA), cryo-coolers, thrusters, solar array drive mechanisms and mobile mirrors, can affect the performance of high pointing accuracy instruments [1-5]. Since these disturbances are significant in the $1 \mathrm{k} \mathrm{Hz}$ frequency range [6] and excite flexible modes of the spacecraft, they cannot be controlled/reduced by the attitude and orbit control systems. Minimising micro-vibrations is a significant problem for spacecraft with high stability requirements, for example the Hubble Space Telescope, GOES-N, GOCE and Hinode (Solar-B) [2,3,5,12-14]. In practice, although the reduction of the vibration level can be attempted by action at the source(s), receiver(s) and along the vibration path(s) [7], the control (minimisation) of the sources is always crucial in the management of micro-vibrations.

In general, WAs are considered to be the largest micro-vibration source onboard satellites [8-11]. A typical WA consists of a rotating flywheel mounted on a shaft, supported by bearings and driven by a brushless DC motor with the whole assembly encased in a cover. Disturbances from a spinning WA generally arise from four main sources: flywheel mass imbalance, bearing mechanical disturbances, motor drive errors and motor disturbances [15]. Masterson et al. [16-18] have conducted substantial research in the area of empirical and analytical reaction wheel disturbance models, and

\footnotetext{
*Corresponding author at: College of Aerospace and Material Engineering, National University of Defense Technology, No. 47 Yanwachi Street, ChangSha 410073, People's Republic of China. Tel.: + 86073184573186.

E-mail addresses: zhouweiyong@nudt.edu.cn (W.-Y. Zhou), gsa@soton.ac.uk (G.S. Aglietti), zz1e08@soton.ac.uk (Z. Zhang).
} 


\section{Nomenclature}

$a 1, a 2, a 3, a 4$ accelerometer identification number

$A_{F 2 y} \quad$ fundamental harmonic amplitude of the force $F_{2 y}$

$A_{M 2 x}$ fundamental harmonic amplitude of the moment $M_{2 x}$

$c_{s x}, c_{s y}, c_{s z}, c_{s \alpha}, c_{s \beta}, c_{s \gamma}$ damping coefficient

CoM centre of mass

$\mathbf{C}_{\mathrm{S}} \quad$ damping matrix of the elastic support

$d_{1}, d_{2}, d_{3}, d_{4}$ location of the accelerometer of $1,2,3,4$

$\overrightarrow{\mathbf{e}}_{\mathbf{x}}, \overrightarrow{\mathbf{e}}_{\mathbf{y}} \quad$ unit vector

$\mathbf{f}_{1} \quad$ input force matrix at the mass centre of the flywheel

$\mathbf{f}_{2}$ output force matrix at the mass centre of the seismic mass

$F_{x}, F_{y}, F_{z}$ force at the interface of the WA and the seismic mass

$F_{2 x}, F_{2 y}, F_{2 z}$ force on the CoM of seismic mass

$\mathbf{G}_{12}$ transfer function from $\mathbf{f}_{1}$ to $\mathbf{f}_{2}$

$h_{1} \quad$ distance from CoM of the flywheel to the elastic support point

$h_{2}$ distance from CoM of the seismic mass to the elastic support point

$i \quad$ imaginary unit

$I_{1 x x}, I_{1 y y}, I_{1 z z}$ moment of inertia of the flywheel

$I_{2 x x}, I_{2 y y}, I_{2 z z}$ moment of inertia of the seismic mass

$k_{x}, k_{y}, k_{z}, k_{\alpha}, k_{\beta} k_{\gamma}$ linear and angle elastic modulus

$\mathbf{K}_{s} \quad$ stiffness matrix of the elastic support

$l_{1} \quad$ distance from CoM of extra mass to CoM of the flywheel

$m_{\text {extra }}$ extra mass in the test

$m_{1} \quad$ mass of the flywheel

$m_{2} \quad$ mass of the seismic mass

$\mathbf{M}_{1} \quad$ mass matrix of the flywheel

$\mathbf{M}_{2}$ mass matrix of the seismic mass
$M_{x}, M_{y}, M_{z}$ moment at the interface of the WA and the seismic mass

$M_{2 x}, M_{2 y}, M_{2 z}$ moment on the CoM of seismic mass

$r \quad$ radius of the flywheel

$\overrightarrow{\mathbf{r}}_{\mathbf{b}} \quad$ radius vector

S elastic support point

SMVMS Seismic Micro-Vibration Measurement System

$\mathbf{T}_{s 1}$ force transfer matrix from $S$ to CoM of flywheel

$\mathbf{T}_{s 2} \quad$ force transfer matrix from $S$ to CoM of seismic mass

$\mathbf{T}_{s x 1}$ displacement transfer matrix from CoM of flywheel to $S$

$\mathbf{T}_{s \times 2}$ displacement transfer matrix from CoM of seismic mass to $S$

$U_{s} \quad$ static imbalance of the flywheel

$U_{d} \quad$ dynamic imbalance of the flywheel

$\overrightarrow{\mathbf{v}} \quad$ velocity vector

WA wheel assembly

$\mathbf{x}_{1} \quad$ displacement matrix of the flywheel CoM

$\mathbf{x}_{2}$ displacement matrix of the seismic mass CoM

$\mathbf{x}_{s} \quad$ relative displacement matrix at the support point $S$

\section{Greek letters}

$\overrightarrow{\boldsymbol{\alpha}} \quad$ acceleration vector

$\alpha_{1}, \beta_{1}, \gamma_{1}, \alpha_{s}, \beta_{s}, \gamma_{s}, \alpha_{2}, \beta_{2}, \gamma_{2}$ Euler angle from the transfer order of $1-2-3(x-y-z)$

$\varsigma_{s x}, \varsigma_{s y}, \varsigma_{s z}, \varsigma_{s \alpha}, \varsigma_{s \beta}, \varsigma_{s \gamma}$ damping ratios

$\phi_{s} \quad$ phase of the static imbalance

$\phi_{d} \quad$ phase of the dynamic imbalance

$\omega_{1}, \omega_{2}, \omega_{3}, \omega_{4}, \omega_{5}$ natural frequency

$\vec{\omega} \quad$ angular velocity vector

$\Omega \quad$ angle velocity of the flywheel

$\|$ absolute value

flywheel mass imbalances are considered to be the most significant, with an amplitude proportional to angular speed and a frequency equal to the rotation frequency (fundamental harmonic). Furthermore, Elias and Miller [19] have proposed a coupled disturbances analysis method using dynamic mass measurement techniques. Shigemune, Yoshiaki, and Masahito et al. [20-22] have established a method to measure lower frequency disturbances induced by WAs, which are traditionally difficult to detect, and revealed that the inner dynamics of the WA closely relate to the disturbances.

Typically, the micro-vibrations emitted by a WA are measured with force/moment measurement tables. Masterson et al. [16-18] have analyzed the vibration data obtained from a Kistler 9253 A force/moment table, whose measurement precision is better than $0.01 \mathrm{~N}$; Elias and Miller [19] tested the WA by fixing it on a six-axis JR3 Universal Force-Moment Sensor System. Shigemune, Yoshiaki, and Masahito [20-22] have tested the disturbances by an air-floating disturbance detector, and achieved a higher sensitivity of lower frequency disturbances, with a resolution less than $0.001 \mathrm{~N}$ for $0.1 \mathrm{~Hz}$.

Often WAs with magnetic bearings are employed due to their greatly reduced mechanical noise emission; however, their cost is significantly higher than that of conventional WAs. In this investigation, an internal soft suspension system provided a form of passive control system to minimise the high frequency disturbances emitted by the WA, including noise from the mechanical bearing assembly. As accurate dynamometric platforms are relatively expensive and usually only available in "specialist" labs, this paper implements an approach based on indirect measurement of forces and moments, retrieved by monitoring the dynamics of a seismic mass (onto which the test specimen was mounted) with accelerometers.

Utilising the Seismic Micro-Vibration Measurement System, the performance of a fictitious rigid WA and a flexible WA (implementing a soft suspension) were tested and compared. A Newton-Euler method has been used to create the analytical model of the soft suspension based WA and the test system, and the model was successfully used to analyze the fundamental harmonic disturbances generated by the static and dynamic imbalance of the flywheel. 


\section{Models and methods}

\subsection{The introduction of the WA system}

During launch, a satellite's WAs have to sustain high accelerations and a harsh vibration environment. Conventional WAs are "rigidly" designed with the flywheel supported by mechanical bearings, as shown in Fig. 1(a). In such designs, the shaft, bearings and supporting structure are sized to withstand launch while providing enough stiffness to deliver a resonance frequency that meets the spacecraft requirements (typically the resonance frequency for this type of equipment has to be greater than $100 \mathrm{~Hz}$ ). However, at times, the requirement on the resonance frequency can be waived and the whole motor/flywheel sub-assembly can be mounted on a soft suspension, such as the one shown in Fig. 1(b) [23]. During launch with the associated accelerations, the "floating" flywheel suspension system deflects closing the clearance gap between the flywheel and casing, until the wheel itself contacts the casing. In this condition any further increase of inertia load is directly supported by the casing (rather than going through the suspension system and the bearings). On orbit, the satellite is subjected to zero acceleration and the stiffness of the suspension system restores the flywheel to its neutral position (in the centre of its casing) as shown Fig. 1(b).

There are two major advantages for such a soft suspension system: (i) during launch the flywheel is directly supported by its casing, hence the bearings supporting the shaft are not required to support the high inertial loads of the flywheel (as in a typical rigid design); (ii) during the spacecraft operation on orbit, the suspension system "filters" the higher frequency vibrations created by the flywheel and motor, hence minimising the mechanical disturbances emitted by the WA while operating.

In design of this suspension system, a mathematical model of the disturbances emitted by the WA is useful to trade-off the various design parameters (mainly the stiffness of the suspension) that characterise the design. The development and validation of such a model is described in the subsequent sections.

\subsection{Mathematical model of the WA and SMVMS}

Other researchers have used energy methods (Lagrange) to build the equations of motion for a symmetrical supported WA [16]; however, here the Newton-Euler method is used, as it has the advantage of directly calculating the acceleration response generated by forces and moments, which is the basic principle of the SMVMS testing system. This method can also be easily extended for a more complex situation.

The WA, with its soft suspension system, could be schematically represented as shown in Fig. 2(b), where the WA is mounted on a seismic mass. The flywheel is on the top, characterised by a mass matrix $\mathbf{M}_{1}$, rigidly connected to the point $S$, by a rigid weightless shaft (the mass of the shaft is actually included in that of the flywheel). At the point $S$, the shaft's three translations and rotation are supported by a mechanical element (suspension system) which provides an appropriate value of stiffness. Forces and moments generated at this point are directly proportional to the relative linear and angular displacements and velocities between shaft and supporting structure.

The other parts of the wheel assembly, mainly its base, are considered as rigid and integrated in the mass matrix seismic block $\mathbf{M}_{2}$. The model can be further expanded if there were more elastic supports $\left(S_{2}, S_{3}\right.$, etc.).

The method is implemented in the following steps:

1. Build up the displacements of the shaft (connected with the flywheel as a rigid body) at the point $S$, as functions of the displacements of the flywheel CoM, $\mathbf{x}_{s 1}=\mathbf{T}_{s x 1} \mathbf{x}_{1}$.

2. Build up the displacements of the supporting seismic mass at the point $S$, as functions of the displacements of the seismic mass CoM, $\mathbf{x}_{s 2}=\mathbf{T}_{s \times 2} \mathbf{x}_{2}$.

3. Derive their relative displacement matrix $\mathbf{x}_{s}$ at the support ordinates $S, \mathbf{x}_{s}=\mathbf{T}_{s x 1} \mathbf{x}_{1}-\mathbf{T}_{s x 2} \mathbf{x}_{2}$.

4. Build the relationships between forces (and moments) and the relative displacements and velocities (and rotations) at the support ordinate.

5. Derive the equations of equilibrium (equations of motion) at the CoM of flywheel and seismic mass, respectively.

(a)

(b)

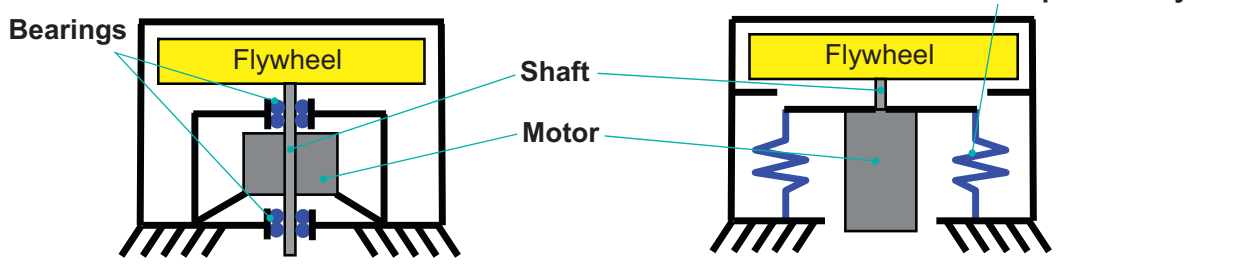

Fig. 1. Schematic view of WAs: (a) conventional bearing based WA and (b) alternative soft spring suspension based WA. 
(a)
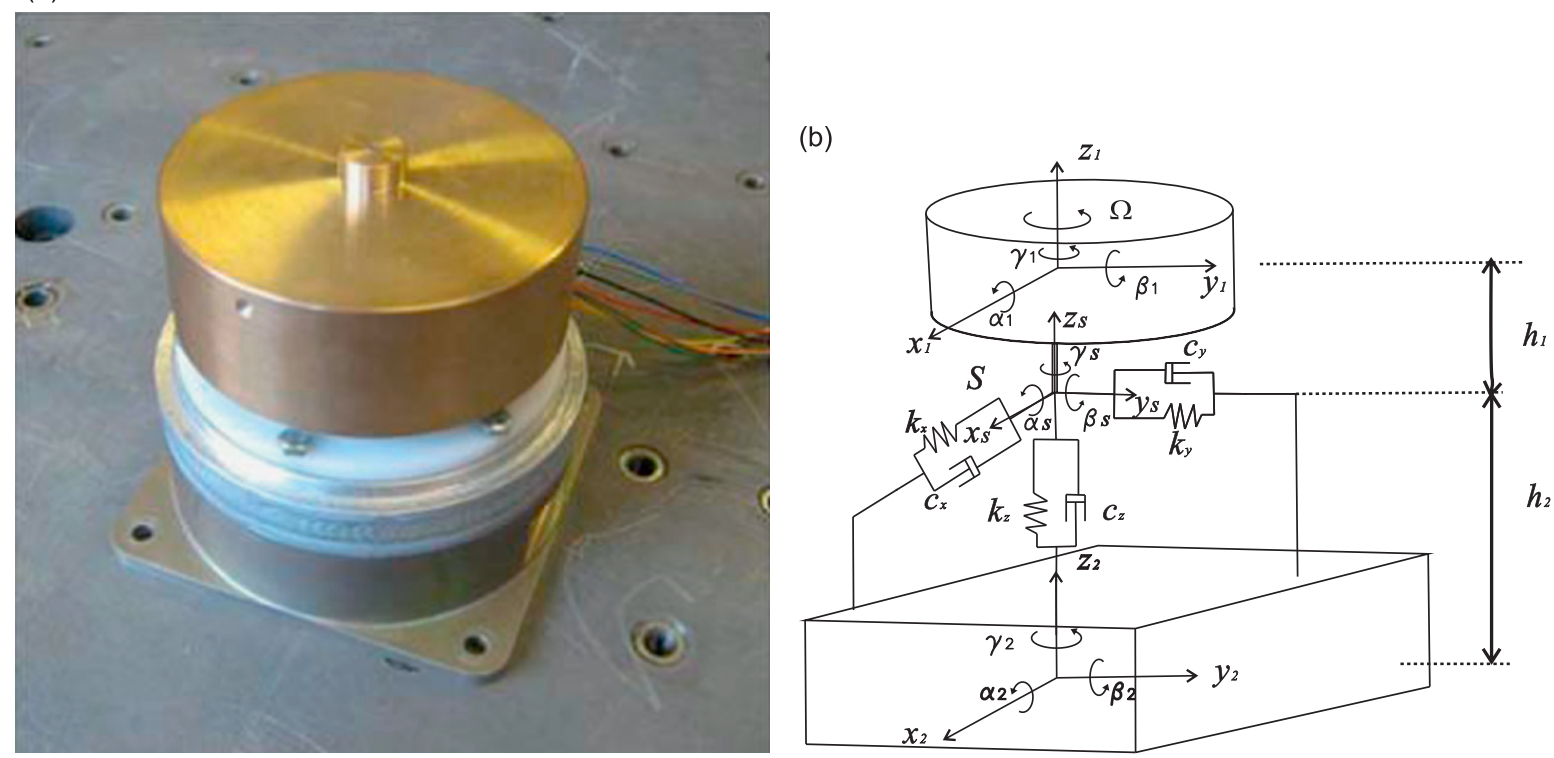

Fig. 2. WA and schematic: (a) WA used in test and (b) schematic of the WA.

Using the method above and the Euler equations [24], ignoring the higher-order terms and considering small angles, (i.e. $\sin \alpha \approx \alpha, \cos \alpha \approx 1$ ), it is possible to obtain the linear equations of motion of the system as

$$
\begin{gathered}
\mathbf{M}_{1} \ddot{\mathbf{x}}_{1}+\mathbf{G} \dot{\mathbf{x}}_{1}+\mathbf{T}_{s 1} \mathbf{C}_{s}\left(\mathbf{T}_{s x 1} \dot{\mathbf{x}}_{1}-\mathbf{T}_{s x 2} \dot{\mathbf{x}}_{2}\right)+\mathbf{T}_{s 1} \mathbf{K}_{s}\left(\mathbf{T}_{s x 1} \mathbf{x}_{1}-\mathbf{T}_{s \times 2} \mathbf{x}_{2}\right)=\mathbf{f}_{1} \\
\mathbf{M}_{2} \ddot{\mathbf{x}}_{2}+\mathbf{T}_{s 2} \mathbf{C}_{s}\left(\mathbf{T}_{s x 2} \dot{\mathbf{x}}_{2}-\mathbf{T}_{s x 1} \dot{\mathbf{x}}_{1}\right)+\mathbf{T}_{s 2} \mathbf{K}_{s}\left(\mathbf{T}_{s x 2} \mathbf{x}_{2}-\mathbf{T}_{s \times 1} \mathbf{x}_{1}\right)=0
\end{gathered}
$$

where $\mathbf{M}_{1}$ and $\mathbf{M}_{2}$ are the mass matrix of the flywheel (including the shaft) and the seismic mass, respectively; $\mathbf{x}_{1}=\left(x_{1}, y_{1}\right.$, $\left.z_{1}, \alpha_{1}, \beta_{1}, \gamma_{1}\right)^{T}$ is the displacement of the flywheel CoM; $\mathbf{x}_{2}=\left(x_{2}, y_{2}, z_{2}, \alpha_{2}, \beta_{2}, \gamma_{2}\right)^{T}$ is the displacement of the seismic mass $\mathrm{CoM}$; note that $\alpha_{j}, \beta_{j}, \gamma_{j}$ are the three Euler angles from the transfer order of 1-2-3; $\mathbf{G}$ is the gyroscopic force matrix; $\mathbf{C}_{s}$ is the damping matrix ( $\mathbf{C}_{s}$ is assumed to be zero, unless explicitly mentioned); $\mathbf{T}_{\mathrm{sxj}}$ is the displacement transfer matrix from the displacement $\mathbf{x}_{j}$ at ordinate location $j$ to the elastic support location $S$; $\mathbf{K}_{\mathrm{s}}$ is the elasticity matrix between relative displacements (and rotation) and forces (and moments) at the support $S$ (suspension system); $\mathbf{T}_{s i}$ is the force transfer matrix from the support point $S$ to the CoM of the part $i$; $\mathbf{f}_{1}$ is the disturbance force input at the flywheel CoM.

The detailed expressions of each of the terms in Eq. (1) can be found in the appendix. In this paper, the rotational speed of the flywheel is constant during each test, so the rotational degree of freedom in the $z$ direction is ignored, meaning we only consider $\mathbf{x}_{1}=\left(x_{1}, y_{1}, z_{1}, \alpha_{1}, \beta_{1}\right)^{T}$ and $\mathbf{x}_{2}=\left(x_{2}, y_{2}, z_{2}, \alpha_{2}, \beta_{2}\right)^{T}$.

Eq.(1) and the method developed above show that once the displacement transfer matrix $\mathbf{T}_{s x j}$ and force transfer matrix $\mathbf{T}_{s i}$ are obtained, it is simple to extend Eq.(1) to a more complex situation; for example, if another elastic support is added to the system.

\subsection{Measurement system}

A series of tests were carried out with the purpose to measure the micro-vibrations emitted by the WA and validate the mathematical model derived in the previous section. Although micro-vibrations emitted by WAs are typically measured using dynamometric platforms (e.g. Kistler table), here the SMVMS described in the previous section was used, therefore the tests also had the purpose validating this measurement system.

As shown in Fig. 3, two accelerometers were attached to the side of an aluminium block (seismic mass) with the WA mounted on the upper surface of the block. The block was then suspended to simulate a "free-free" condition using a pair of elastic supports. In this way, the motion of the block can be considered as that of a rigid body floating in space, under the action of the forces and moments produced by the WA. With the accelerometers attached at appropriate locations, the dynamics of the system can be reconstructed, and the loads produced by the WA can be estimated.

The experimental system can also be schematically modelled as shown in Fig. 2; the flywheel is on the top, with a distance $h_{1}$, from the elastic support point $S$, with stiffness $\mathbf{K}_{s}$; the mass properties of the seismic mass are concentrated at a distance $h_{2}$ below the support point $S$. 
(a)

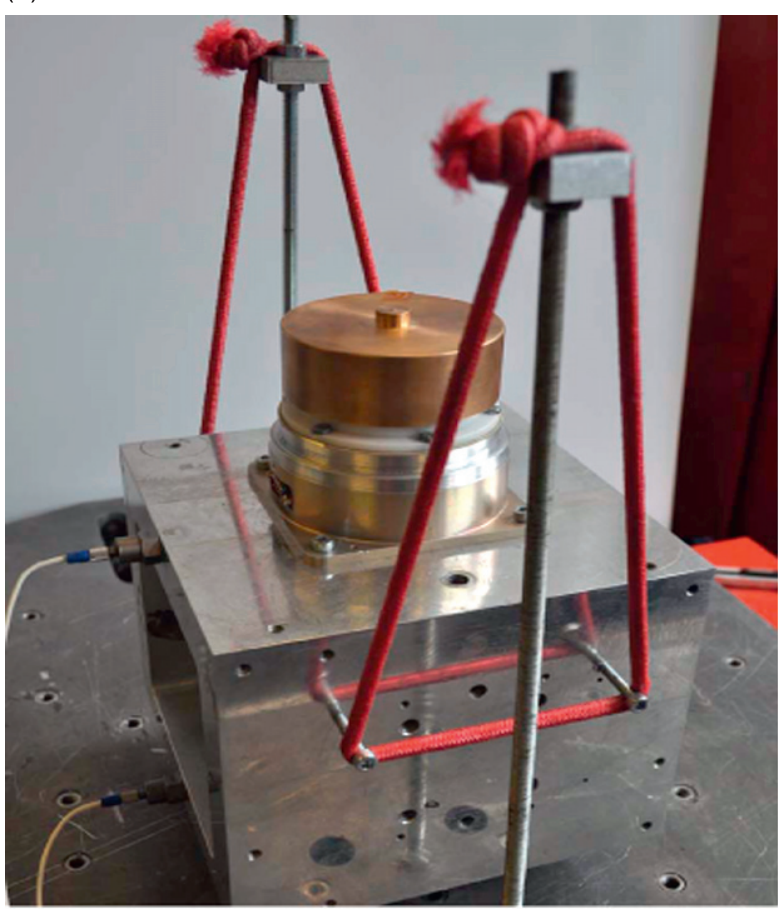

(b)

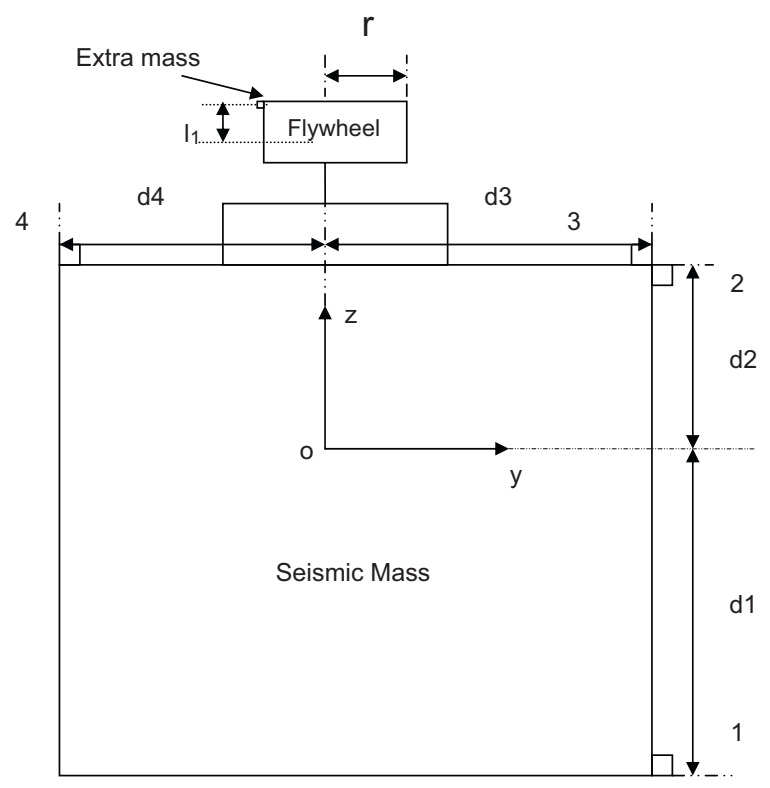

Fig. 3. Testing system and schematic: (a) SMVMS testing system and (b) schematic of the system.

\subsubsection{The acceleration of test point}

Fig. 3 shows the locations of the accelerometers of the test system. The in-plane rotations and displacements (in the $y$ direction) are detected using signals from the acceleormeters $a 1$ and $a 2 . d_{1}, d_{2}$ are the respective vertical distances from the accelerometer locations 1 and 2 to the CoM of seismic block; $d_{3}, d_{4}$ are the respective horizontal distances from the accelerometer locations 3 and 4 to the CoM of seismic block. The acceleration at the accelerometer locations can be expressed as

$$
\overrightarrow{\mathbf{a}}=\frac{\mathbf{d} \overrightarrow{\mathbf{v}}}{\mathbf{d t}}+\frac{\mathbf{d} \overrightarrow{\boldsymbol{\omega}}}{\mathbf{d t}} \times \overrightarrow{\mathbf{r}}_{b}+\overrightarrow{\boldsymbol{\omega}} \times\left(\vec{\omega} \times \overrightarrow{\mathbf{r}}_{b}\right)
$$

where $\overrightarrow{\mathbf{r}}_{b}$ is the radius vector from the CoM of the seismic mass to the accelerometers; $\overrightarrow{\mathbf{v}}$ and $\overrightarrow{\boldsymbol{\omega}}$ are the velocity vector and angular velocity vector of the seismic block, respectively. Assuming $\overrightarrow{\boldsymbol{\omega}}$ is small, the centripetal acceleration term $\overrightarrow{\boldsymbol{\omega}} \times(\overrightarrow{\boldsymbol{\omega}} \times \overrightarrow{\mathbf{r}})$ is much smaller than the terms $\mathbf{d} \overrightarrow{\mathbf{v}} / \mathbf{d t}$ and $(\mathbf{d} \overrightarrow{\boldsymbol{\omega}} / \mathbf{d t}) \times \overrightarrow{\mathbf{r}}$, thus can be ignored. If the ordinate axes are the inertia axes, then the accelerations of locations 1 and 2 in Fig. 3(b) can be expressed as

$$
\left[\begin{array}{l}
a_{1} \\
a_{2}
\end{array}\right]=\left[\begin{array}{cc}
\frac{1}{m_{2}} & \frac{d_{1}}{I_{2 x x}} \\
\frac{1}{m_{2}} & -\frac{d_{2}}{I_{2 x x}}
\end{array}\right]\left[\begin{array}{c}
F_{2 y} \\
M_{2 x}
\end{array}\right]
$$

where $F_{2 y}$ and $M_{2 x}$ are the force and moment applied on the CoM of the seismic mass, respectively.

From Eq. (3), the following equation can be obtained:

$$
\left[\begin{array}{c}
F_{2 y} \\
M_{2 x}
\end{array}\right]=\left[\begin{array}{cc}
\frac{m_{2} d_{2}}{d_{1}+d_{2}} & \frac{m_{2} d_{1}}{d_{1}+d_{2}} \\
\frac{I_{2 x x}}{d_{1}+d_{2}} & -\frac{I_{2 x x}}{d_{1}+d_{2}}
\end{array}\right]\left[\begin{array}{l}
a_{1} \\
a_{2}
\end{array}\right]
$$

Knowing the accelerations $a_{1}$ and $a_{2}$, using the signals of the accelerometers (whose sensitivities are 1001 and $988 \mathrm{mV} / \mathrm{g}$, respectively), it is possible to deduce the force and the moment at the CoM of the seismic block. Thus, it is possible to calculate the force $F_{y}$ and moment $M_{x}$ applied at the interface of the seismic block by the WA:

$$
\left[\begin{array}{c}
F_{y} \\
M_{x}
\end{array}\right]=\left[\begin{array}{cc}
\frac{m_{2} d_{2}}{d_{1}+d_{2}} & \frac{m_{2} d_{1}}{d_{1}+d_{2}} \\
\frac{I_{2 x x}+m_{2} d_{2}^{2}}{d_{1}+d_{2}} & \frac{-I_{2 x x}+m_{2} d_{1}^{2}}{d_{1}+d_{2}}
\end{array}\right]\left[\begin{array}{l}
a_{1} \\
a_{2}
\end{array}\right]
$$


Assuming a symmetric system in the axial direction, it is possible to describe the amplitudes of force $F_{x}$ and moment $M_{y}$ at the interface as

$$
\begin{aligned}
& \left|F_{x}\right|=\left|F_{y}\right| \\
& \left|M_{y}\right|=\left|M_{x}\right|
\end{aligned}
$$

Two accelerometers are enough to calculate the radial disturbances (force and moment) at the interface between the WA and seismic block.

Using another pair of accelerometers (locations 3 and 4 in Fig. 3), it is then possible to describe the expression as

$$
\left[\begin{array}{l}
a_{3} \\
a_{4}
\end{array}\right]=\left[\begin{array}{cc}
\frac{1}{m_{2}} & \frac{d_{3}}{I_{2 x x}} \\
\frac{1}{m_{2}} & -\frac{d_{4}}{I_{2 x x}}
\end{array}\right]\left[\begin{array}{c}
F_{2 z} \\
M_{2 x}
\end{array}\right]
$$

This allows the axial disturbance force $F_{2 z}$ to be obtained, and further validate the value of the moment obtained by accelerometers 1 and 2:

$$
\left[\begin{array}{c}
F_{2 z} \\
M_{2 x}^{\prime}
\end{array}\right]=\left[\begin{array}{cc}
\frac{m_{2}}{2} & \frac{m_{2}}{2} \\
\frac{I_{2 x x}}{2 d_{3}} & -\frac{I_{2 x x}}{2 d_{3}}
\end{array}\right]\left[\begin{array}{l}
a_{3} \\
a_{4}
\end{array}\right]
$$

where $M_{2 x}^{\prime}$ is the moment at the CoM of the seismic mass computed by $a 3$ and $a 4$, and here $d_{3}=d_{4}$.

\subsubsection{Test procedures}

The tests were performed to evaluate the performance (in terms of micro-vibration emission) of the soft suspension based WA and compare it with a conventional rigid design.

The tests were carried out in two different configurations:

(a) WA in its nominal state (i.e. soft suspension system).

(b) WA with internal suspension system blocked, to simulate a rigid design.

The tests were carried out in the following sequences:

(1) Using miniature accelerometers on the flywheel, a "tap test" was conducted to retrieve the resonance frequencies of the WA at rest. In each direction, the WA behaves as a sdof (single degree of freedom), therefore, this test allowed verification of the stiffness of the suspension system given knowledge of the inertia.

(2) An extra imbalance mass of 1-g was attached to the edge of the flywheel, with the WA mounted on the seismic block, as shown in Fig. 3(b).

(3) An initial data acquisition was carried out with the WA at rest, to verify the background noise threshold.

(4) The WA (with 1-g unbalancing mass) was powered and controlled to constant speed, starting from 100 to 2000 rev/ min in steps of $100 \mathrm{rev} / \mathrm{min}$, with 5-min at each step. This known level of imbalance of the flywheel allowed validation of the mathematical model.

(5) The imbalance mass was removed and the WA ran from 100 to $7800 \mathrm{rev} / \mathrm{min}$, in intervals of $100 \mathrm{rev} / \mathrm{min}$ at 5-min time intervals, to investigate the steady-state performance of the WA at different speeds.

(6) The locations of accelerometers 1 and 2 were changed to the new locations 3 and 4 , to obtain the force in the axial direction and test the moment of the previous test.

The sequences above were repeated for both configurations, i.e. soft suspension flexible and conventional rigid design, where the latter was reproduced by locking the soft suspension system inside the WA.

\section{Experimental results and analysis}

\subsection{The tap test results}

Table 1 shows the three natural frequencies of the flexible WA and the rigid WA at rest, respectively retrieved from the tests. A detailed discussion of the three modes (radial rocking, radial translation and axial translation) of the WA can be found in reference [15].

Table 1

Natural frequencies of the flexible and rigid WAs.

\begin{tabular}{lcr}
\hline WA type & Radial translation $(\mathrm{Hz})$ & Radial rocking $(\mathrm{Hz})$ \\
\hline Flexible & 52.1 & 25.3 \\
Rigid & 224.2 & 65.0 \\
\hline
\end{tabular}




\subsection{Natural frequencies}

With the WA at rest $(\Omega=0)$, the resonance frequencies are available from the tap tests, allowing verification of the stiffness of the supporting springs, $\mathbf{K}_{s}$. Solving the characteristic equation of Eq. (1), the natural frequencies can be obtained. Fig. 4 shows the Campbell diagrams of the flexible WA grounded (i.e. with the seismic mass blocked) and with the seismic mass free, and compares the results with the Finite Element Analysis (FEA).

With reference to Fig. 4(a), there are two pairs of backward and forward whirl curves. One pair is $\omega_{1}$ and $\omega_{2}$. $\omega_{1}$ is the backward whirl curve, decreasing with the speed of the flywheel; $\omega_{2}$ is the forward whirl curve, increasing with rotational speed. The other pair is $\omega_{4}$ and $\omega_{5}$. The backward whirl curve, $\omega_{4}$, decreases with rotational speed, very close to the other forward curve $\omega_{2}$ when the rotational speed is greater than $3000 \mathrm{rev} / \mathrm{min}$. The forward whirl curve, $\omega_{5}$ increases with flywheel's rotational speed. The axial translation natural frequency $\omega_{3}$, is approximately $45.6 \mathrm{~Hz}$, irrespective of the rotational speed, since the axial equation is decoupled from the others in the mathematical model. Since the flywheel is not symmetrical supported $\left(h_{1} \neq 0\right)$, the radial rocking model and radial translation model are coupled with each other. It is impossible to separate them on the curves, and the mode can transform from one type to another. For example, $\omega_{2}$ is most like radial rocking mode at low rotational speed, and become more and more like radial translation mode with the increasing rotational speed.

The coupling of the WA and the seismic mass increased the natural frequencies beyond that of the fixed WA, as shown in Fig. 4(b). The seismic block altered the behaviours of the WA slightly. Fig. 4 also shows that the theoretical results agree closely with the FEA results, confirming the validity of the mathematical model of Eq. (1).

\subsection{Accuracy estimate and fundamental harmonics}

\subsubsection{Background noise}

Fig. 5 shows the background noise of $a 1$ when the WA was at rest; $a 2$ was a similar level, and hence is omitted here.

Fig. 5 shows that the background noise of the accelerometer $a 1$ is below $0.0001 \mathrm{~m} / \mathrm{s}^{2}$ at the frequency range $2-10 \mathrm{~Hz}$, and is below $0.00005 \mathrm{~m} / \mathrm{s}^{2}$ above $10 \mathrm{~Hz}$. From Eq. (5), the level of noise corresponding to a force at the interface is approximately $0.001 \mathrm{~N}$ (or moment about $0.0001 \mathrm{~N} \mathrm{~m}$ ) at the frequency 2-10 Hz, and $0.0005 \mathrm{~N}$ (or moment $0.00005 \mathrm{~N}$ m) between 10 and $1000 \mathrm{~Hz}$. The high output at low frequency ( $<2 \mathrm{~Hz}$ ) is due to the rigid body motion of the set up (i.e. the seismic mass supported by elastic supports).
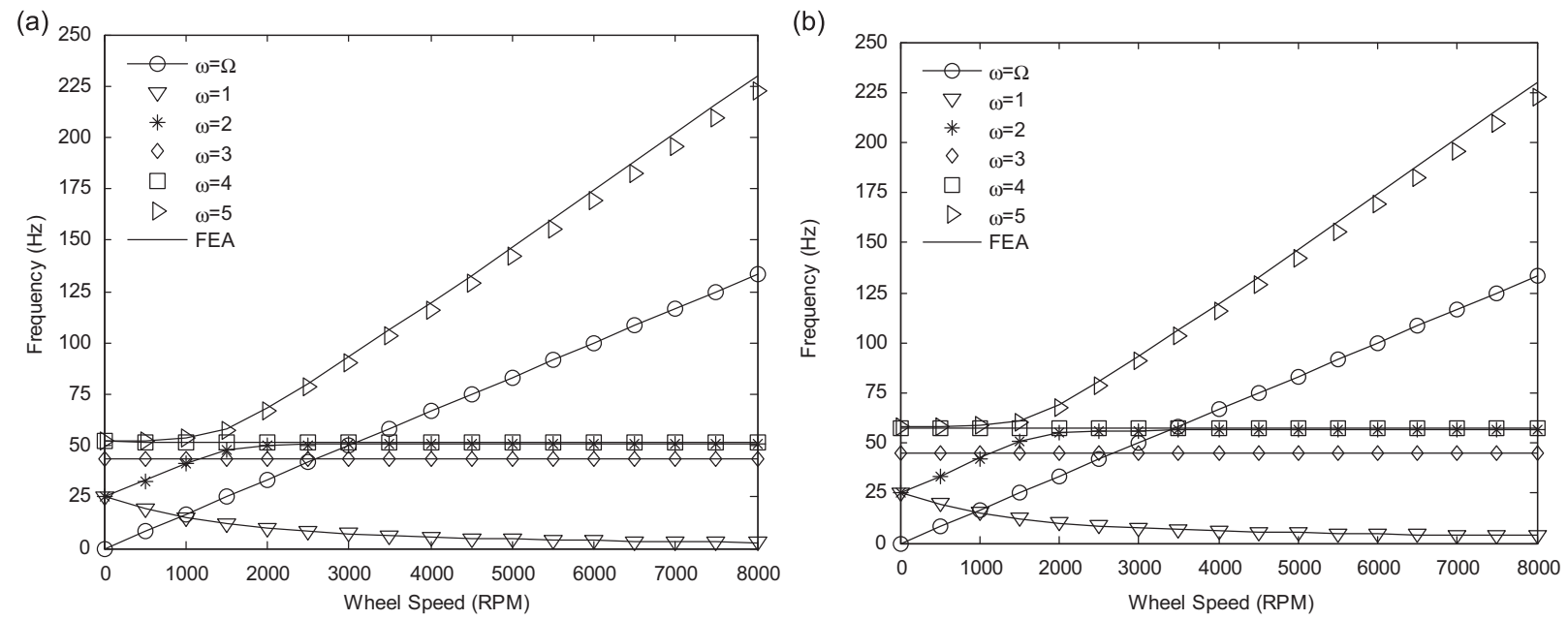

Fig. 4. Campbell diagrams (theoretical and FEA results): (a) grounded WA Campbell diagram and (b) WA with seismic mass system Campbell diagram.

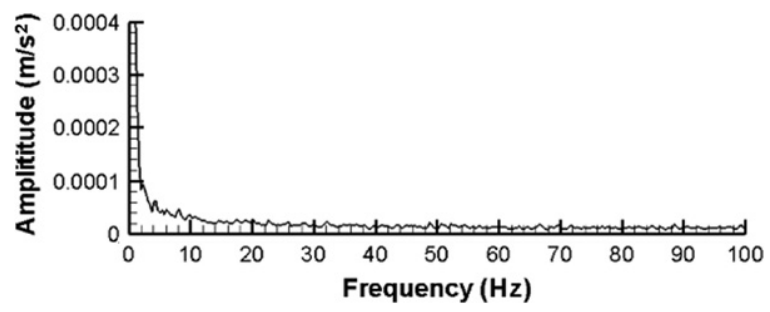

Fig. 5. Measurement background noise for accelerometer $a 1$. 


\subsubsection{Emissions during WA operations}

Fig. 6 shows the frequency response of acceleration at the location $a 1$ for the WA with soft suspension and for the rigid WA. The amplitude of $a 2$ was close to $a 1$ and is omitted.

From Eqs. (3) to (8), the forces and moments can be deduced from accelerations measured by the testing system, so the test sensitivity depends on the mass property of the seismic mass and the locations of the accelerometers. The heavier the seismic block, the more the results approach those obtained using a dynamometric table; however this is limited by the sensitivity of the accelerometers (which have to detect the very small movements of the mass) and noise. The mass of the seismic block utilised in this work $(10 \mathrm{~kg})$, and the sensitivity of the accelerometers $(\sim 1000 \mathrm{mV} / \mathrm{g})$ allowed performances comparable to dynamometric tables.

Fig. 6(a) also shows that for the flexible WA, at a speed of $300 \mathrm{rev} / \mathrm{min}$, besides the disturbance at $5 \mathrm{~Hz}$ (fundamental harmonic), there is a small peak in frequency close to $60 \mathrm{~Hz}$, which corresponds with the whirl curves $\omega_{4}$ and $\omega_{5}$ in Fig. 4(b), for the rigid WA, Fig. 6(c) shows there is no such extra disturbance at $60 \mathrm{~Hz}$. Fig. 6(d) alternatively shows that there are relatively large disturbances between 200 and $400 \mathrm{~Hz}$.

\subsubsection{Fundamental harmonic response at the WA interface}

Fig. 7 shows comparisons of the force and moment fundamental harmonic response between the flexible WA and the rigid WA.

(a)

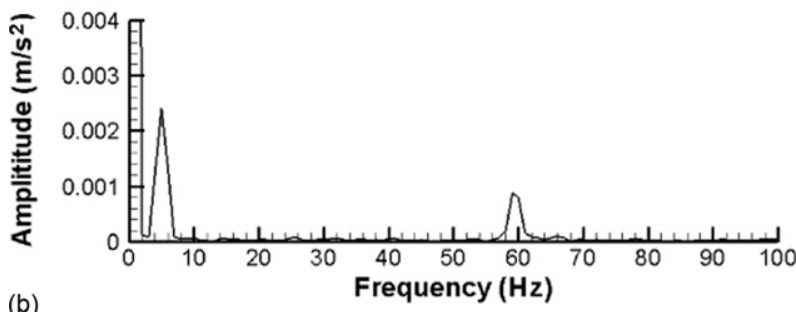

(b)

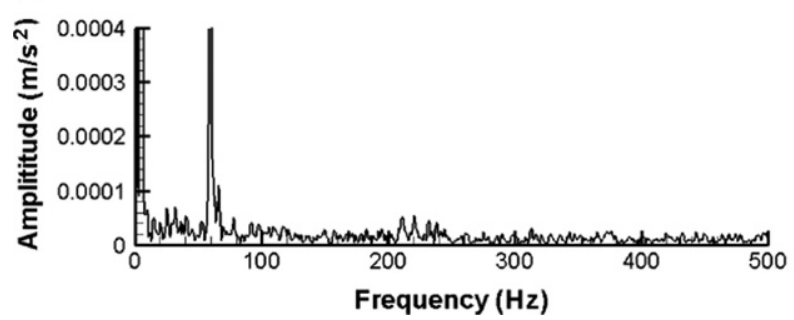

(c)

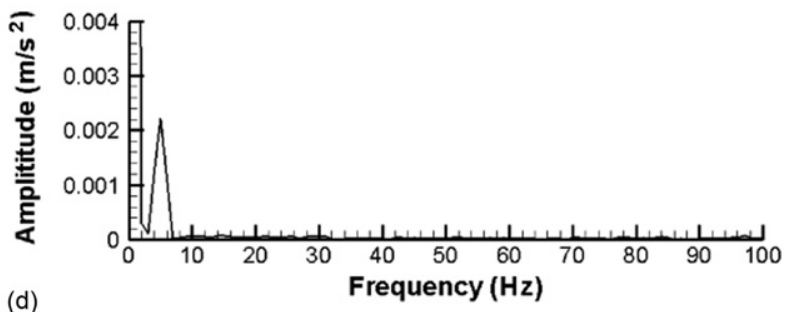

(d)

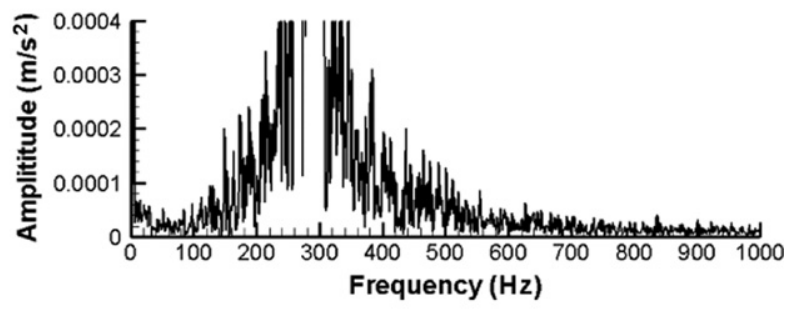

Fig. 6. Acceleration amplitude of $a 1$ for a flexible and rigid WA: (a) flexible WA $300 \mathrm{rev} / \mathrm{min}$; (b) flexible WA $300 \mathrm{rev} / \mathrm{min}$ (scaled); (c) rigid WA $300 \mathrm{rev} / \mathrm{min}$; and (d) rigid WA $300 \mathrm{rev} / \mathrm{min}$ (scaled).

(a)

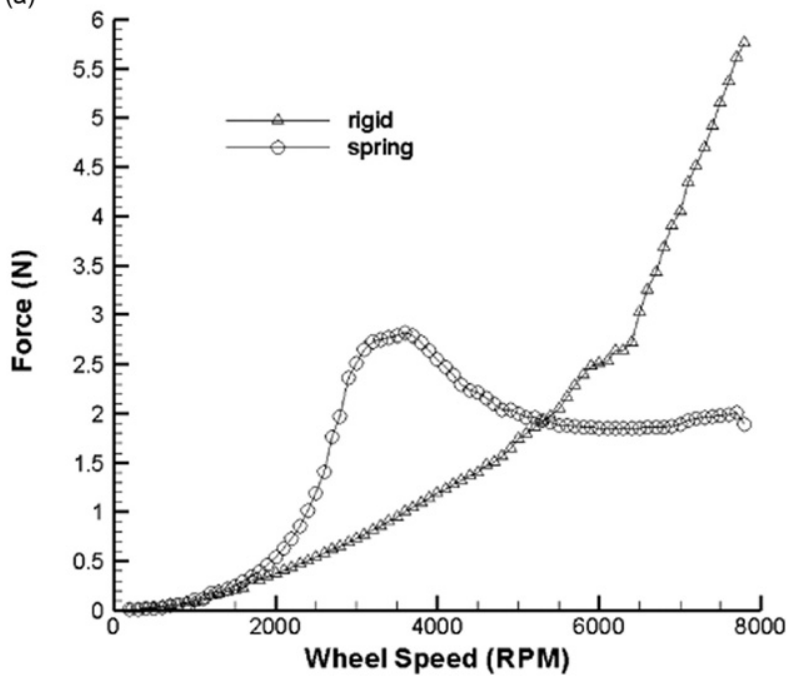

(b)

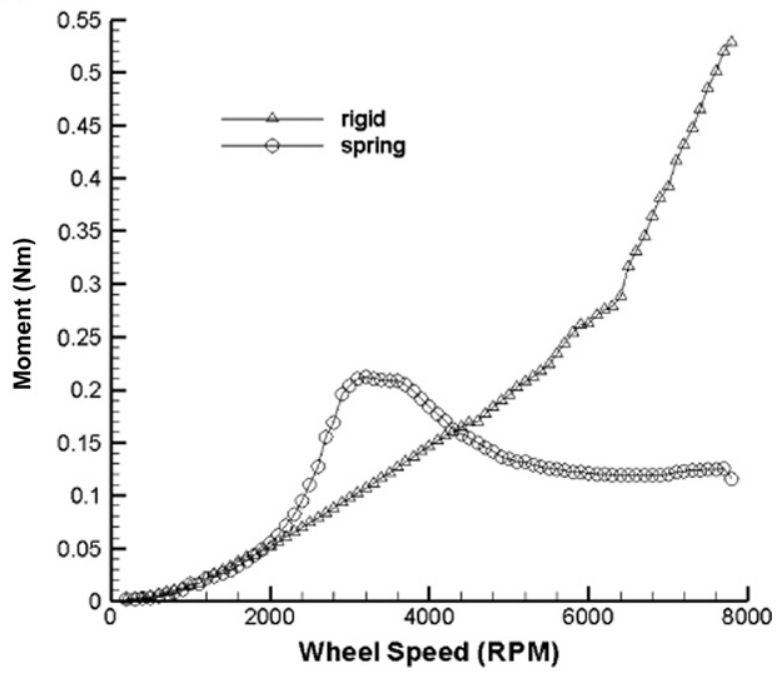

Fig. 7. Disturbances at the WA interface (experimental results): (a) Fundamental harmonic force and (b) Fundamental harmonic moment. 
A resonance range in the region of $2700-3600 \mathrm{rev} / \mathrm{min}(45-60 \mathrm{~Hz})$ is shown, whereas for the rigid model, no resonance is excited in the test speed range, and the resonance amplitude increases proportional to the square of rotational speed.

For the flexible WA, the amplitudes of both radial force and moment initially increase with speeds and reach their first resonance peak at approximately $2700 \mathrm{rev} / \mathrm{min}(45 \mathrm{~Hz})$, which is the intersection point of the fundamental harmonic $\omega=\Omega$ and the axial natural frequency $\omega_{3}$ in Fig. $4(\mathrm{~b})$. After this point, they increase slowly until they reach a second resonance peak at approximately $3600 \mathrm{rev} / \mathrm{min}(60 \mathrm{~Hz})$, nearing the intersection point of the fundamental harmonic $\omega=\Omega$ and the first forward whirl curve $\omega_{2}$ in Fig. 4(b). As the two resonance frequencies are exceeded, the disturbance force input still increases with rotational speed, though the force and moment decrease. According to Fig. 4(b), there is an intersection point of the fundamental harmonic and the first backward whirl $\omega_{1}$, however with no clear peak as shown in Fig. 7. This is because the response of the WA in our test is mainly influenced by the mass imbalance, which would primarily induce the forward whirl.

For the rigid WA, the amplitudes of the force and moment curves follow parabolas, since the disturbance forces generated by the mass imbalance are proportional to the square of the rotational speed. The advantage of the soft suspension based WA is a reduction in disturbance at the higher frequency band, after the rotation speed passes the resonance frequency range. The disadvantage is that the disturbance forces are relatively large when nearing the resonance frequency. One solution is to increase the damping in the supporting spring to diminish the peak value; another could be to use a softer spring to support the flywheel, to further lower the resonance frequencies.

\subsection{Vertical force and lateral moment check}

Fig. 8 shows the moment $M_{2 x}^{\prime}$ (the moment at the CoM of the seismic mass) and the force $F_{z}$, calculated using results from the accelerometers $a 3$ and $a 4$.

Fig. 8(a) shows that the moment computed by $a 3$ and $a 4$ compares well with the moment by $a 1$ and $a 2$ in the majority of the WA speed range. However, there are some differences in the speed range $1500-4000 \mathrm{rev} / \mathrm{min}$. These are due to the wire of the motor, which is discussed in 3.6.

As shown in Fig. 8(b), the axial harmonic force is relatively low compared with the radial force in Fig. 8(a), and the peak of the axial force is near to $2700 \mathrm{rev} / \mathrm{min}$, which is the axial translation natural frequency of the system $\left(\omega_{3}=45.6 \mathrm{~Hz}\right.$ in Fig. $\left.4(\mathrm{~b})\right)$.

\subsection{WA interface reactions}

Fig. 9 shows waterfall plots of the radial force and moment at the interface between the WA and seismic mass.

Fig. 9(a)-(f) clearly shows the fundamental, second and higher harmonic disturbances of the flexible WA. The second and higher harmonics of the flexible WA reflect the system nonlinearity, and are mainly caused by the nonlinearity and coupling of the elastic support (which were considered linear and decoupled in the mathematical model). The second and higher harmonics are much lower than the fundamental harmonic, hence the fundamental harmonic is the main disturbance and is modelled and analyzed in part 4.

Fig. 9(e) also shows that there is a small disturbance line at the frequency of approximately $60 \mathrm{~Hz}$, which corresponds with the relative whirl curves in Fig. 4(b).

Fig. 9(e) and (f) shows that the higher frequency disturbances of the flexible WA are greatly reduced when compared with the rigid WA, at the frequencies of $200-400 \mathrm{~Hz}$ in particular.

(a)

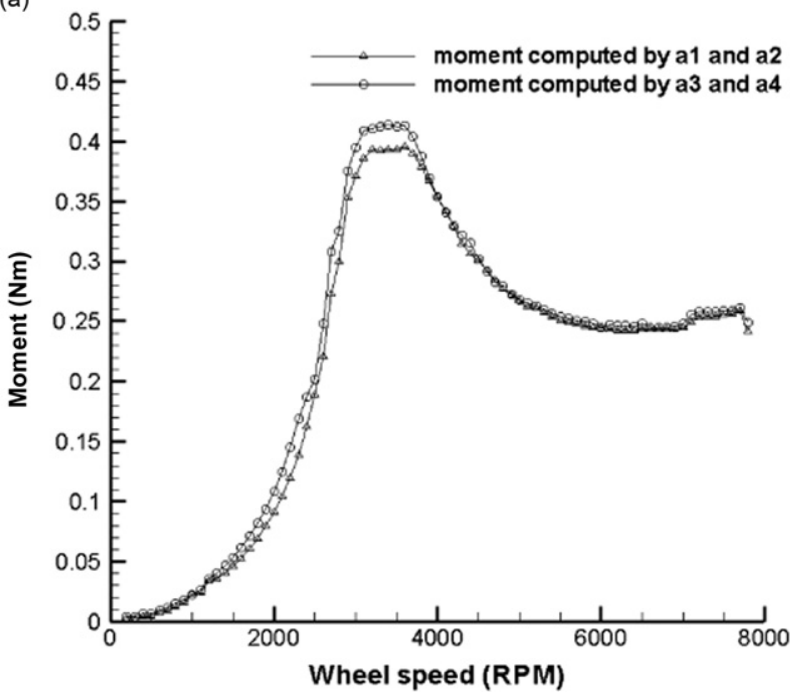

(b)

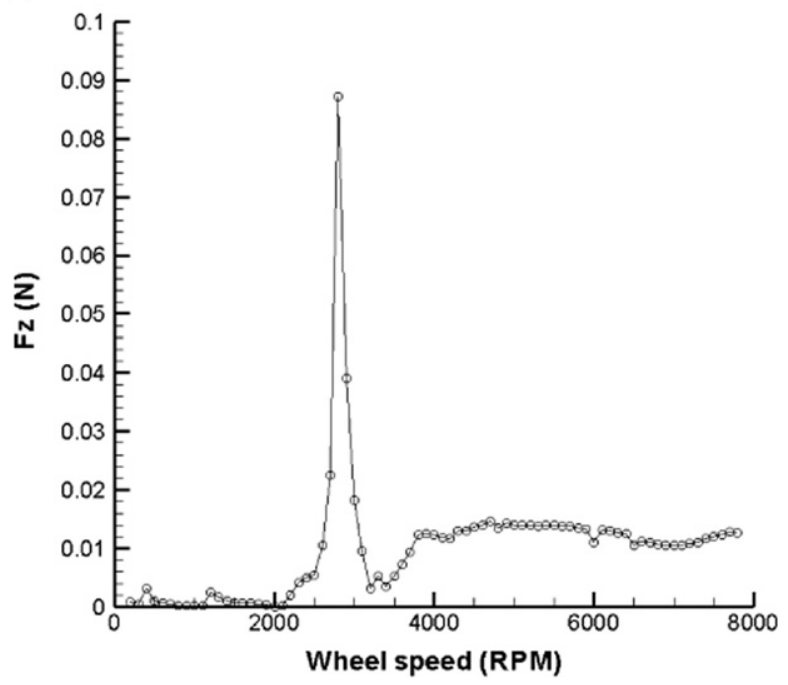

Fig. 8. Disturbances from $a 3$ and $a 4$ (experimental results): (a) fundamental harmonic of moment and (b) fundamental harmonic of force. 
(a)

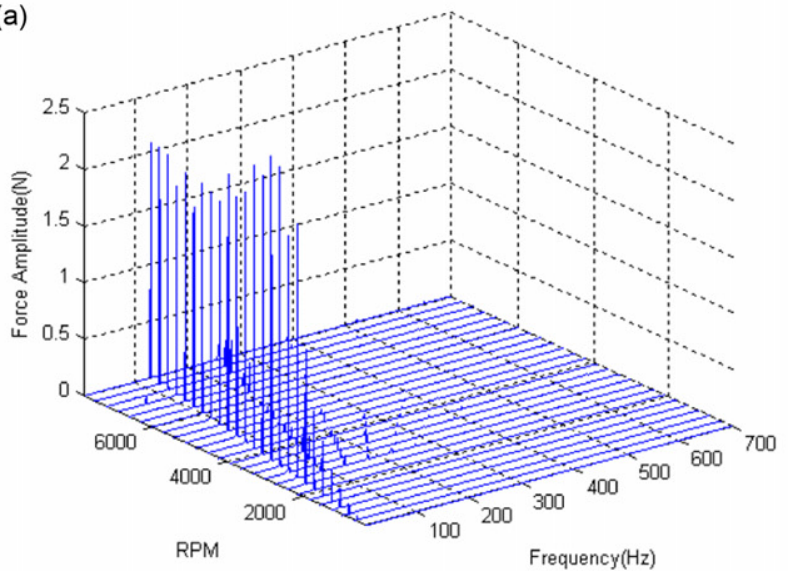

(c)

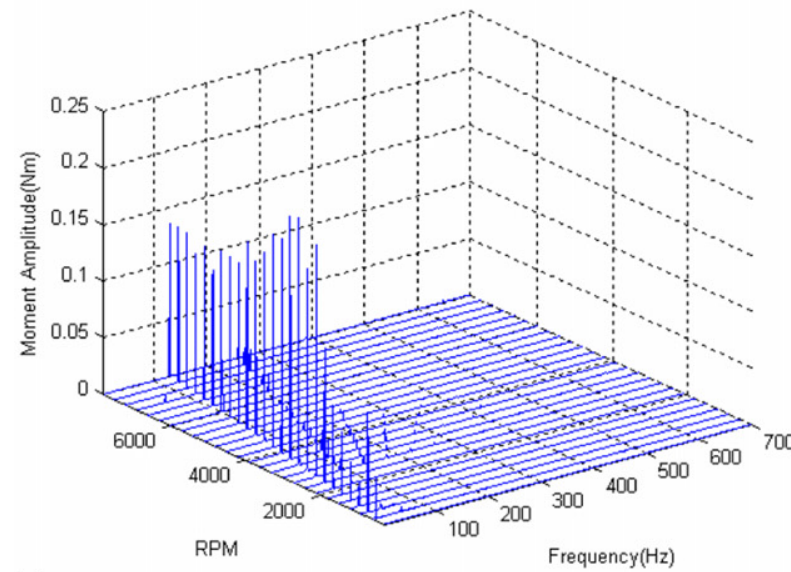

(e)

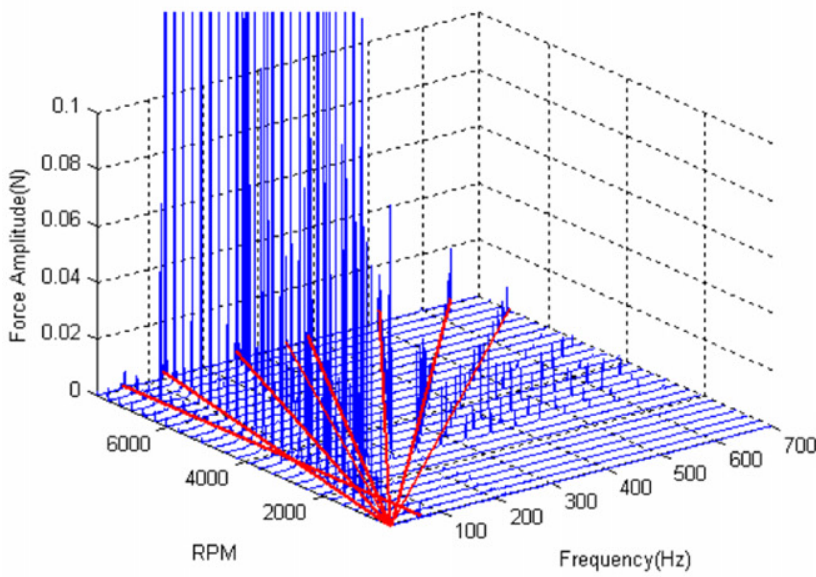

(b)

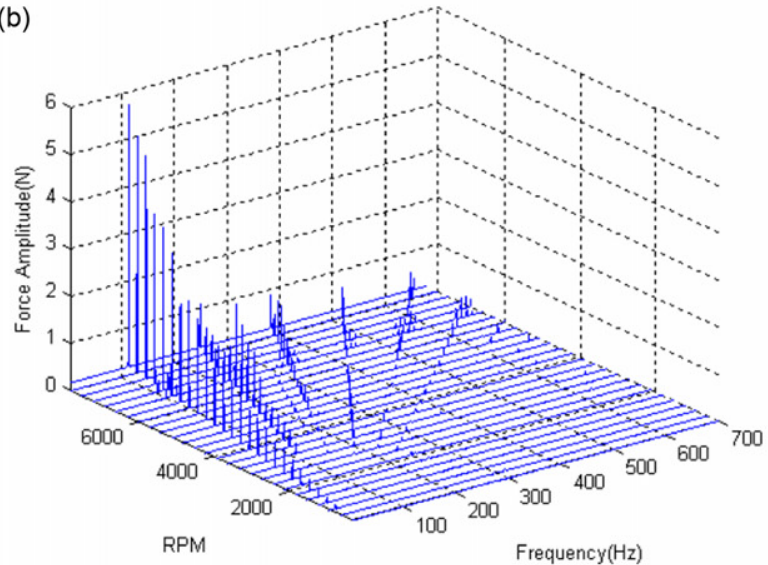

(d)

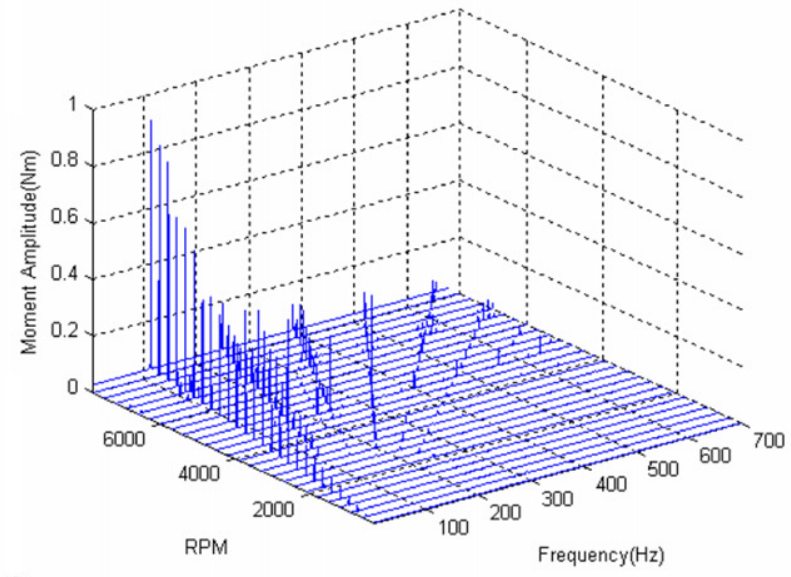

(f)

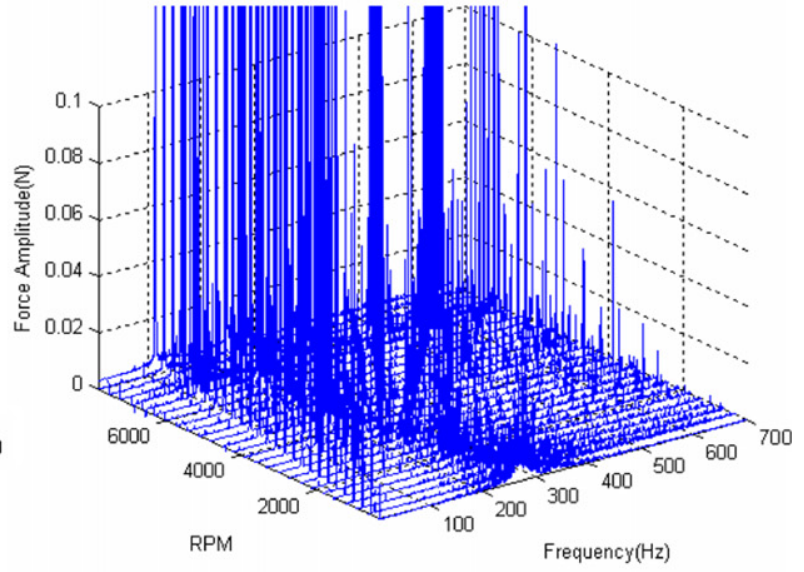

Fig. 9. Waterfall plot at the interface (experimental results): (a) force of the flexible WA; (b) force of the rigid WA; (c) moment of the flexible WA; (d) moment of the rigid WA; (e) force of the flexible WA (scaled); and (f) force of the rigid WA (scaled).

\subsection{Test repeatability}

For the WA with soft suspension, there were small differences between sets of results in the same test condition, especially near resonance frequencies between 45 and $60 \mathrm{~Hz}$. This phenomenon was caused by the electrical wires of the motor, which act as a load path during the test. As the wires were bundled, the difference became more obvious. Though there were some minor differences even between test repeats when the wire configuration remained the same, however 
the differences were never more than $8 \%$ at any frequency or rotational speed. Clearly the effects of the electrical wires of the motor should be considered in the future WA design.

\subsection{Comments on the SMVMS}

This work has shown that SMVMS has achieved relatively high measurement accuracy utilising simple equipment, and with the advantage, with respect to dynamometric platforms, that the seismic mass is elastically suspended, thus filtering the ground noise at medium to high frequencies. However, there are also several limitations: firstly, due to the seismic platform modes at frequencies $<1 \mathrm{~Hz}$, this system does not detect accurately the low frequency disturbances (e.g. below $2 \mathrm{~Hz}$ ). Secondly, inaccuracy of the physical parameters of the system, such as $m_{2}, I_{2 x x}, d_{1}, d_{2}, d_{3}, d_{4}$, and the sensitivity of the accelerometers directly influence the accuracy of the results. In this work, masses and dimensions were directly measured to a high accuracy. From there the density was determined and the moment of inertial calculated from a SolidWorks model, verified by hand calculations. Thirdly, the disturbance forces obtained at the interface by the SMVMS are representative of the "coupled" system, WA and the seismic mass, which also makes the WA's resonant frequencies higher than those of the rigidly grounded WA.

\section{Numerical simulation and analysis}

\subsection{Transfer function}

Taking the Laplace transform of Eq. (1) and supposing $\mathbf{x}_{1}(0)=\dot{\mathbf{x}}_{1}(0)=\mathbf{x}_{2}(0)=\dot{\mathbf{x}}_{2}(0)=0$, the following can be obtained:

$$
\left[\begin{array}{cc}
s^{2} \mathbf{M}_{1}+s \mathbf{G}+\mathbf{T}_{s 1}\left(s \mathbf{C}_{s}+\mathbf{K}_{s}\right) \mathbf{T}_{s \times 1} & -\mathbf{T}_{s 1}\left(s \mathbf{C}_{s}+\mathbf{K}_{s}\right) \mathbf{T}_{s \times 2} \\
-\mathbf{T}_{s 2}\left(s \mathbf{C}_{s}+\mathbf{K}_{s}\right) \mathbf{T}_{s \times 1} & s^{2} \mathbf{M}_{2}+\mathbf{T}_{s 2}\left(s \mathbf{C}_{s}+\mathbf{K}_{s}\right) \mathbf{T}_{s \times 2}
\end{array}\right]\left[\begin{array}{c}
\mathbf{X}_{1} \\
\mathbf{X}_{2}
\end{array}\right]=\left[\begin{array}{c}
\mathbf{f}_{1} \\
0
\end{array}\right]
$$

The matrix in the expression above can be simply written as

$$
\left[\begin{array}{cc}
s^{2} \mathbf{M}_{1}+s \mathbf{G}+\mathbf{T}_{s 1}\left(s \mathbf{C}_{s}+\mathbf{K}_{s}\right) \mathbf{T}_{s 1 \times 1} & -\mathbf{T}_{s 1}\left(s \mathbf{C}_{s}+\mathbf{K}_{s}\right) \mathbf{T}_{s x 2} \\
-\mathbf{T}_{s 2}\left(s \mathbf{C}_{s}+\mathbf{K}_{s}\right) \mathbf{T}_{s x 1} & s^{2} \mathbf{M}_{2}+\mathbf{T}_{s 2}\left(s \mathbf{C}_{s}+\mathbf{K}_{s}\right) \mathbf{T}_{s 1 \times 2}
\end{array}\right]=\left[\begin{array}{cc}
\mathbf{A} & \mathbf{B} \\
\mathbf{C} & \mathbf{D}
\end{array}\right]
$$

Assuming $\mathbf{D}$ can be inverted, it is possible to obtain $\mathbf{x}_{2}=-\mathbf{D}^{-1} \mathbf{C} \mathbf{x}_{1}$, and, if $\left(\mathbf{A}-\mathbf{B D}{ }^{-1} \mathbf{C}\right)$ can be inverted, we are able to write $\mathbf{x}_{1}=\left(\mathbf{A}-\mathbf{B D}^{-1} \mathbf{C}\right)^{-1} \mathbf{f}_{1}$. Finally it is possible to obtain the transfer function between the disturbance $\mathbf{f}_{1}$ and the force $\mathbf{f}_{2}$ (with $\mathbf{f}_{2}$ being the force acting on the CoM of seismic mass):

$$
\begin{gathered}
\mathbf{f}_{2}=\mathbf{G}_{12} \mathbf{f}_{1} \\
\mathbf{G}_{12}=\mathbf{T}_{s 2}\left(s \mathbf{C}_{s}+\mathbf{K}_{s}\right)\left(\mathbf{T}_{s \times 2} \mathbf{D}^{-1} \mathbf{C}+\mathbf{T}_{s x 1}\right)\left(\mathbf{A}-\mathbf{B D}^{-1} \mathbf{C}\right)^{-1}
\end{gathered}
$$

Taking the input disturbances $\mathbf{f}_{1}$ as an input, and the force acting on the seismic mass $\mathbf{f}_{2}$ as an output, the system can be simplified as an "input-output" linear system, and $\mathbf{G}_{12}$ is the transfer function.

\subsection{Static imbalance and dynamic imbalance}

Though the disturbances of the WA are of various origins: flywheel mass imbalance, bearing mechanical noise, motor driven errors and motor disturbances; the fundamental harmonic disturbances are mainly due to static and dynamic imbalances [15], which can be represented as shown in Fig. 10.

The force generated by the static imbalance is given as

$$
F_{r}=m_{s} r \Omega^{2}=U_{s} \Omega^{2}
$$

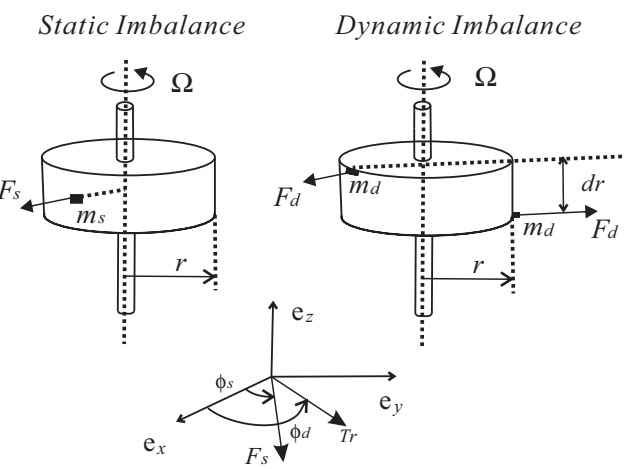

Fig. 10. Static imbalance and dynamic imbalance. 
where the static imbalance is reproduced, considering a mass $m_{s}$, at the distance $r$ from the axis (note that the static imbalance mass, and zero width space; $U_{s}=m_{s} r$ is a flywheel mass property). The force can be expressed as

$$
\begin{array}{r}
\overrightarrow{\mathbf{F}}_{\mathbf{x}}(t)=U_{s} \Omega^{2} \cos \left(\Omega t+\phi_{s}\right) \cdot \overrightarrow{\mathbf{e}}_{\mathbf{x}} \\
\overrightarrow{\mathbf{F}}_{\mathbf{y}}(t)=U_{s} \Omega^{2} \sin \left(\Omega t+\phi_{s}\right) \cdot \overrightarrow{\mathbf{e}}_{\mathbf{y}}
\end{array}
$$

where $\phi_{s}$ is the phase of the force, $\overrightarrow{\mathbf{e}}_{\mathbf{x}}$ and $\overrightarrow{\mathbf{e}}_{\mathbf{y}}$ are the unit vectors.

Similarly, the moment generated by dynamic imbalance is given as

$$
T_{r}=m_{d} r d \Omega^{2}=U_{d} \Omega^{2}
$$

where $m_{d}$ is the dynamic imbalance mass, and $U_{d}=m_{d} r d_{r}$ is a flywheel property. The force due to the dynamic imbalance can therefore be written as

$$
\begin{aligned}
& \overrightarrow{\mathbf{T}}_{\mathbf{x}}(t)=U_{d} \Omega^{2} \cos \left(\Omega t+\phi_{d}\right) \cdot \overrightarrow{\mathbf{e}}_{\mathbf{x}} \\
& \overrightarrow{\mathbf{T}}_{\mathbf{y}}(t)=U_{d} \Omega^{2} \sin \left(\Omega t+\phi_{d}\right) \cdot \overrightarrow{\mathbf{e}}_{\mathbf{y}}
\end{aligned}
$$

where $\phi_{d}$ is the phase of the moment caused by the dynamic imbalance. Details of the disturbances of WAs can be found in Ref. [19]. In general, the $m_{s}$ and $m_{d}$ are small enough to be ignored in the mass matrix of the flywheel in Eq. (1).

Taking the static imbalance and dynamic imbalance as an input,

$$
\mathbf{f}_{\mathbf{1}}(t)=\left[\begin{array}{lllll}
U_{s} \Omega^{2} \cos \left(\Omega t+\phi_{s}\right) & U_{s} \Omega^{2} \sin \left(\Omega t+\phi_{s}\right) & 0 & U_{d} \Omega^{2} \cos \left(\Omega t+\phi_{d}\right) & U_{d} \Omega^{2} \sin \left(\Omega t+\phi_{d}\right)
\end{array}\right]^{T},
$$

the steady-state output of $F_{2 y}$ and $M_{2 x}$ can be expressed as:

$$
\begin{gathered}
F_{2 y}=A_{F 2 y} \cdot \sin \left(\Omega t+\angle G_{F 2 y}\right) \\
M_{2 x}=A_{M 2 x} \cdot \sin \left(\Omega t+\angle G_{M 2 x}\right) \\
A_{F 2 y}=\left|G_{F 2 y}\right| \\
A_{M 2 x}=\left|G_{M 2 x}\right| \\
G_{F 2 y}=\left[\begin{array}{lllll}
0 & 1 & 0 & 0 & 0
\end{array}\right] \mathbf{G}_{12}(\Omega j)\left[\begin{array}{c}
U_{s} \Omega^{2}\left(i \cos \phi_{s}-\sin \phi_{s}\right) \\
U_{s} \Omega^{2}\left(\cos \phi_{s}+i \sin \phi_{s}\right) \\
0 \\
U_{d} \Omega^{2}\left(i \cos \phi_{d}-\sin \phi_{d}\right) \\
U_{d} \Omega^{2}\left(\cos \phi_{d}+i \sin \phi_{d}\right)
\end{array}\right] \\
G_{M 2 x}=\left[\begin{array}{lllll}
0 & 0 & 0 & 1 & 0
\end{array}\right] \mathbf{G}_{12}(\Omega j) \\
{\left[\begin{array}{c}
U_{s} \Omega^{2}\left(i \cos \phi_{s}-\sin \phi_{s}\right) \\
U_{s} \Omega^{2}\left(\cos \phi_{s}+i \sin \phi_{s}\right) \\
0 \\
U_{d} \Omega^{2}\left(i \cos \phi_{d}-\sin \phi_{d}\right) \\
U_{d} \Omega^{2}\left(\cos \phi_{d}+i \sin \phi_{d}\right)
\end{array}\right]}
\end{gathered}
$$

where $A_{F 2 y}$ and $A_{M 2 x}$ are the amplitudes of $F_{2 y}$ and $M_{2 x}$ respectively.

\subsection{Results comparison}

To test the model, an extra mass of $m_{\text {extra }}=1.00 \mathrm{~g}$ was attached to the upper edge of the wheel, as shown in Fig. 3(b), to produce known disturbances of static imbalance and dynamic imbalance simultaneously, which can be expressed as follows:

$$
U_{s}=m_{\mathrm{extra}} r, \quad \phi_{s}=-\frac{\pi}{2}, \quad U_{d}=m_{\mathrm{extra}} r l_{1}, \quad \phi_{d}=0
$$

where $l_{1}$ is the vertical distance from the extra mass to the CoM of the flywheel (as shown in Fig. 3(b)). Assuming that $\phi_{s}=-\pi / 2, \phi_{d}=0$, the static imbalance mass and the dynamic imbalance mass would lie in the same axial cross section of the flywheel.

Due to manufacturing tolerances, even without extra masses on the flywheel, there is an imbalance that produces acceleration $a_{0}$. Adding the extra mass, the resulting acceleration should be bounded between the limits $a_{\min }$ and $a_{\max }$, where $a \in\left[a_{\min }, a_{\max }\right],\left|a_{\min }\right|=\left|a_{\text {mextra }}\right|-\left|a_{0}\right|$, and $\left|a_{\max }\right|=\left|a_{\text {mextra }}\right|+\left|a_{0}\right| . a_{\text {mextra }}$ is the acceleration caused by the extra $1 \mathrm{~g}$ of mass deduced by the mathematical method.

Fig. 11 shows the comparisons of the test results, theoretical results and FEA results of the fundamental harmonic amplitude of accelerations (the lower bound is $a_{\min }$ and the upper bound is $a_{\max }$ ). The theoretical results match well with the test results, within the upper and lower bounds, which confirms that the mathematical model is reasonably accurate. 
(a)

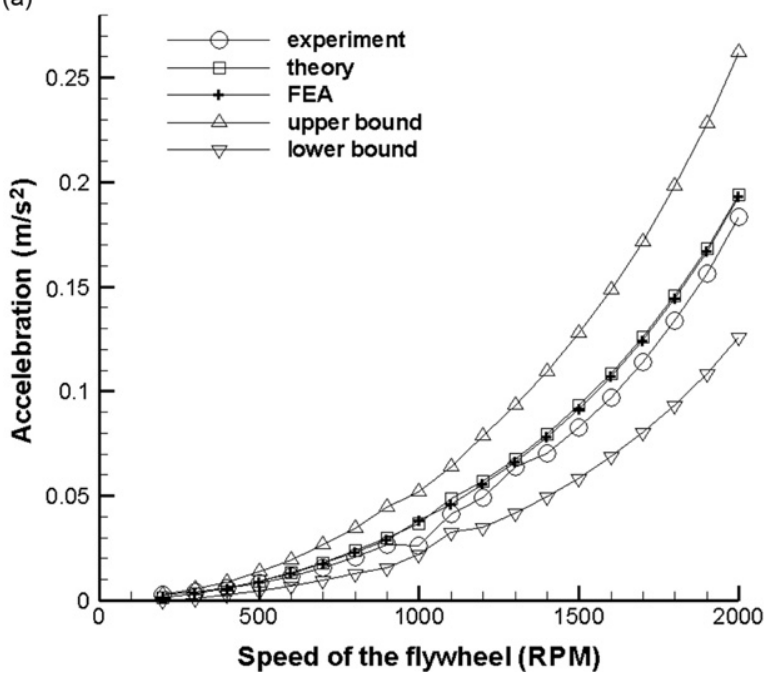

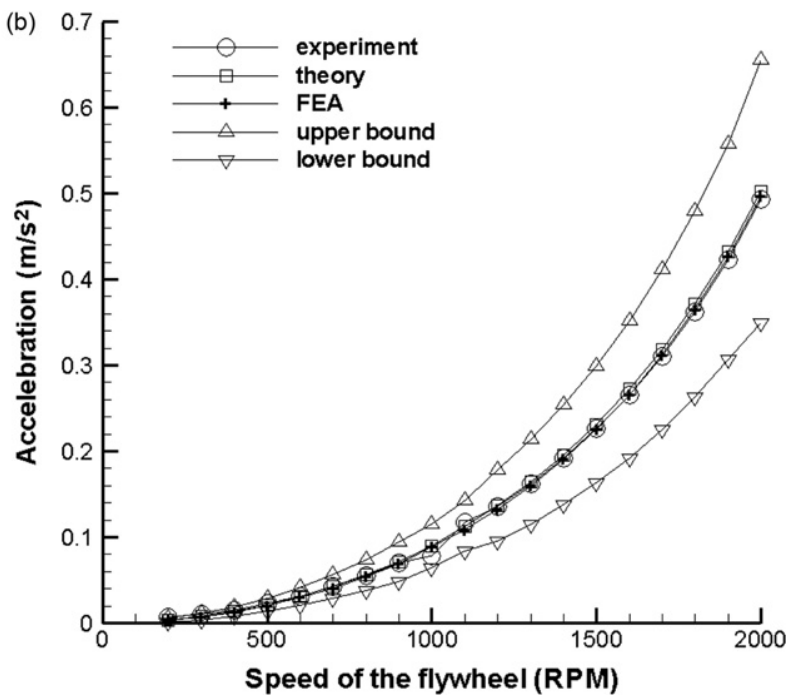

Fig. 11. Fundamental harmonic acceleration amplitude test comparison: (a) acceleration amplitude of $a 1$ and (b) acceleration amplitude of $a 2$.

\subsection{Static and dynamic imbalances estimate}

Eq. (16) shows that the amplitude of output depends on following input parameters: rotational speed $\Omega$, static imbalance $U_{s}$ and dynamic imbalance $U_{d}$ and their phases. However, if one of the imbalances (static or dynamic) is supposed to coincide with the $x$ direction, only the phase difference between them needs to be considered in the equation. At the same time, if the $m_{s}$ and $m_{d}$ are supposed in the same cross section of the flywheel (the phase difference is known), it is possible to estimate the imbalance of the flywheel by the test results. Supposing $\phi_{s}=-\pi / 2, \phi_{d}=0$, the amplitude of $F_{2 y}$ and $M_{2 x}$ can be expressed as

$$
\begin{aligned}
& A_{F 2 y}=\left|\left[\begin{array}{lllll}
0 & 1 & 0 & 0 & 0
\end{array}\right] \mathbf{G}_{12}(\Omega j)\left[\begin{array}{lllll}
U_{s} \Omega^{2} & -U_{s} \Omega^{2} i & 0 & U_{d} \Omega^{2} i & U_{d} \Omega^{2}
\end{array}\right]^{T}\right|
\end{aligned}
$$

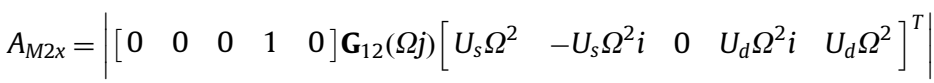

Using the equations above, the static imbalance $U_{s}$ and dynamic imbalance $U_{d}$ at $1500 \mathrm{rev} / \mathrm{min}$ can be deduced from the test results as follows: $U_{s}=6.9 \times 10^{-6} \mathrm{~kg} \mathrm{~m}, U_{d}=5.474 \times 10^{-7} \mathrm{~kg} \mathrm{~m}$; knowing $U_{s}$ and $U_{d}$, the damping matrix can be added and changed to "fit" the response of the acceleration at the resonance frequency of $3600 \mathrm{rev} / \mathrm{min}$. Then it can be plotted the fundamental harmonic amplitude of $a 1$ and $a 2$, as shown in Fig. 12(a). The damping ratios here are $\varsigma_{s x}=\frac{c_{s x}}{2 \sqrt{k_{x} m_{1}}}=\varsigma_{1 y}=\frac{c_{s y}}{2 \sqrt{k_{y} m_{1}}}=\varsigma_{s \theta}=\frac{c_{s \theta}}{2 \sqrt{k_{\theta} I_{1 x x}}}=\varsigma_{s \phi}=0.165$.

For the rigid support WAs, when the static imbalance and dynamic imbalance mass are the same, the fundamental harmonic amplitude of $a 1$ and $a 2$ are as shown in Fig. 12(b).

Fig. 12 shows that the theoretical estimated results of the accelerations match well with the test ones, verifying the validity of the transfer function. Fig. 12 indicates that if the flywheel mass properties (static imbalance and dynamic imbalance) are obtained, the mathematical model can effectively represent the fundamental harmonic of the WA. As mentioned earlier, Fig. 9(a) shows that the fundamental harmonic is the primary disturbance; this mathematical model can therefore be used to estimate the main effects produced by a WA.

\section{Conclusion}

This article has described the mathematical modelling and testing of a soft spring suspension based wheel assembly (WA) for satellite applications. A Newton-Euler method has been used to develop the mathematical model of the WA including the fundamental harmonic disturbance resulting from the flywheel imbalance. This model could be further expanded to represent the case of a WA mounted on a flexible structure (e.g. a spacecraft panel).

A Seismic Micro-Vibration Measurement System (SMVMS) which indirectly computes forces and moments produced at the WA attachment interface, from the accelerations of a seismic block, has been introduced to test the WA. An advantage of this type of measurement, with respect to dynamometric platforms, is that the seismic mass is elastically suspended thus filtering the ground noise at medium to high frequencies. This work has shown that this relatively simple test system achieves high resolution $(0.001 \mathrm{~N}$ in $2-10 \mathrm{~Hz}$, and $0.0005 \mathrm{~N}$ in $10-1000 \mathrm{~Hz})$, and clearly shows the performances in terms of micro-vibrations emitted by the WAs. 

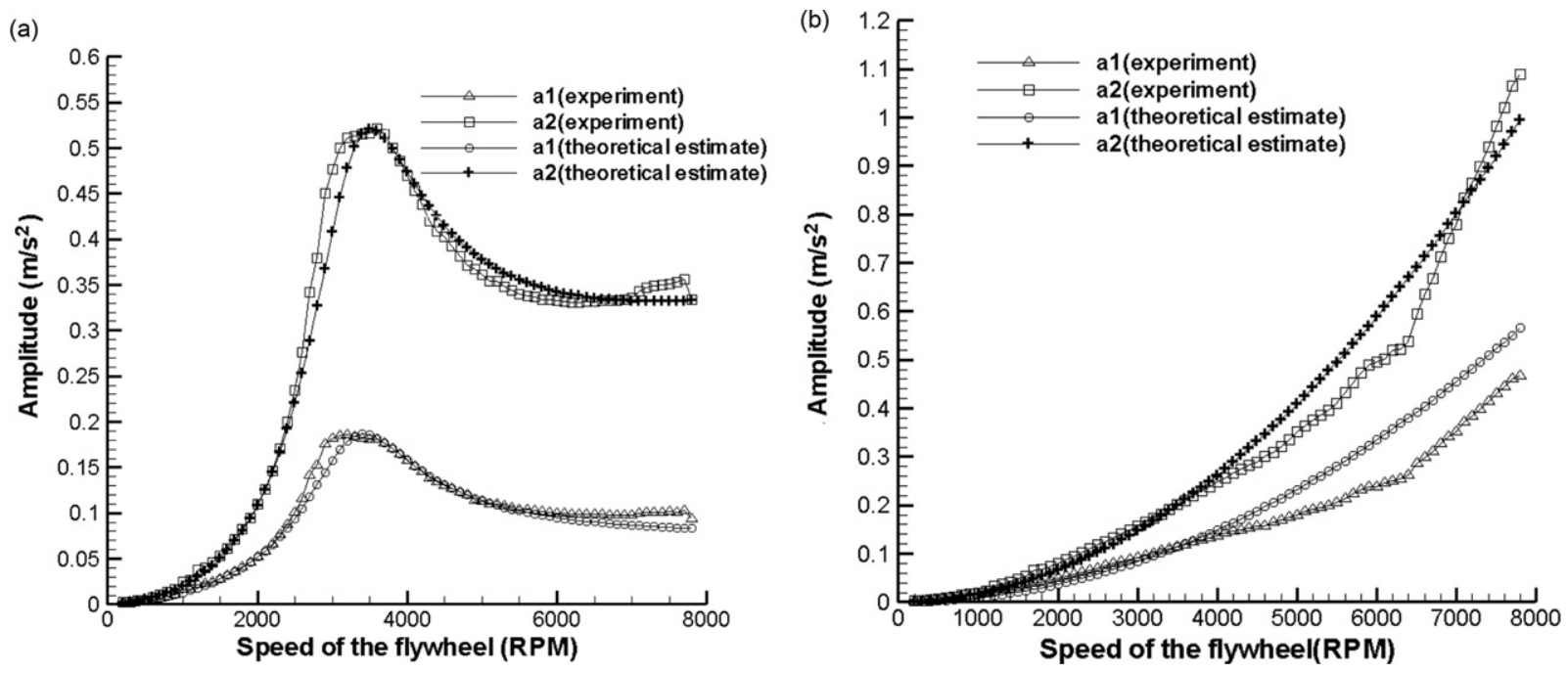

Fig. 12. Fundamental harmonic accelerations comparison: (a) fundamental harmonic of flexible WA and (b) fundamental harmonic of rigid WA.

A fictitious conventional rigid WA design (here referred to as rigid WA) has been tested, together with the WA implementing soft suspension using this SMVMS. The rigid design is showed to produce considerable vibration in the frequency range $200-400 \mathrm{~Hz}$, which is difficult to model and to control. Alternatively, the soft suspension system, acting like a mechanical filter, considerably reduces high frequency vibrations and its micro-vibration emissions (which are mainly of a low frequency) can be modelled simply by considering the flywheel mass imbalance (without the necessity to model phenomena such as bearing noise). Therefore, the model only accounts for the fundamental harmonic and its amplification due to the WA resonance, as this is considerably more important, in terms of interface loads, than the second and higher harmonics which are mainly filtered by the suspension system.

\section{Acknowledgement}

The authors would like to acknowledge the support of Satellite Services Ltd. throughout this work.

\section{Appendix}

$$
\begin{aligned}
\mathbf{M}_{1}=\left[\begin{array}{cccccc}
m_{1} & 0 & 0 & 0 & 0 & 0 \\
0 & m_{1} & 0 & 0 & 0 & 0 \\
0 & 0 & m_{1} & 0 & 0 & 0 \\
0 & 0 & 0 & I_{1 x x} & 0 & 0 \\
0 & 0 & 0 & 0 & I_{1 y y} & 0 \\
0 & 0 & 0 & 0 & 0 & I_{1 z z}
\end{array}\right], \quad \mathbf{M}_{2}=\left[\begin{array}{cccccc}
m_{2} & 0 & 0 & 0 & 0 & 0 \\
0 & m_{2} & 0 & 0 & 0 & 0 \\
0 & 0 & m_{2} & 0 & 0 & 0 \\
0 & 0 & 0 & I_{2 x x} & 0 & 0 \\
0 & 0 & 0 & 0 & I_{2 y y} & 0 \\
0 & 0 & 0 & 0 & 0 & I_{2 z z}
\end{array}\right] \\
\mathbf{T}_{s x 1}=\left[\begin{array}{cccccc}
1 & 0 & 0 & 0 & -h_{1} & 0 \\
0 & 1 & 0 & h_{1} & 0 & 0 \\
0 & 0 & 1 & 0 & 0 & 0 \\
0 & 0 & 0 & 1 & 0 & 0 \\
0 & 0 & 0 & 0 & 1 & 0 \\
0 & 0 & 0 & 0 & 0 & 1
\end{array}\right], \quad \mathbf{T}_{s x 2}=\left[\begin{array}{cccccc}
1 & 0 & 0 & 0 & h_{2} & 0 \\
0 & 1 & 0 & -h_{2} & 0 & 0 \\
0 & 0 & 1 & 0 & 0 & 0 \\
0 & 0 & 0 & 1 & 0 & 0 \\
0 & 0 & 0 & 0 & 1 & 0 \\
0 & 0 & 0 & 0 & 0 & 1
\end{array}\right] \\
\mathbf{K}_{s}=\left[\begin{array}{cccccc}
k_{x} & 0 & 0 & 0 & 0 & 0 \\
0 & k_{y} & 0 & 0 & 0 & 0 \\
0 & 0 & k_{z} & 0 & 0 & 0 \\
0 & 0 & 0 & k_{\alpha} & 0 & 0 \\
0 & 0 & 0 & 0 & k_{\beta} & 0 \\
0 & 0 & 0 & 0 & 0 & k_{\gamma}
\end{array}\right], \quad \mathbf{G}=\left[\begin{array}{cccccc}
0 & 0 & 0 & 0 & 0 & 0 \\
0 & 0 & 0 & 0 & 0 & 0 \\
0 & 0 & 0 & 0 & 0 & 0 \\
0 & 0 & 0 & 0 & \Omega I_{1 z z} & 0 \\
0 & 0 & 0 & -\Omega I_{1 z z} & 0 & 0 \\
0 & 0 & 0 & 0 & 0 & 0
\end{array}\right]
\end{aligned}
$$




$$
\mathbf{C}_{s}=\left[\begin{array}{cccccc}
c_{s x} & 0 & 0 & 0 & 0 & 0 \\
0 & c_{s y} & 0 & 0 & 0 & 0 \\
0 & 0 & c_{s z} & 0 & 0 & 0 \\
0 & 0 & 0 & c_{s \alpha} & 0 & 0 \\
0 & 0 & 0 & 0 & c_{s \beta} & 0 \\
0 & 0 & 0 & 0 & 0 & c_{s \gamma}
\end{array}\right], \quad \mathbf{T}_{s 1}=\left[\begin{array}{cccccc}
1 & 0 & 0 & 0 & 0 & 0 \\
0 & 1 & 0 & 0 & 0 & 0 \\
0 & 0 & 1 & 0 & 0 & 0 \\
0 & h_{1} & 0 & 1 & 0 & 0 \\
-h_{1} & 0 & 0 & 0 & 1 & 0 \\
0 & 0 & 0 & 0 & 0 & 1
\end{array}\right], \quad \mathbf{T}_{s 2}=\left[\begin{array}{cccccc}
1 & 0 & 0 & 0 & 0 & 0 \\
0 & 1 & 0 & 0 & 0 & 0 \\
0 & 0 & 1 & 0 & 0 & 0 \\
0 & -h_{2} & 0 & 1 & 0 & 0 \\
h_{2} & 0 & 0 & 0 & 1 & 0 \\
0 & 0 & 0 & 0 & 0 & 1
\end{array}\right]
$$

\section{References}

[1] U. Roberto, M. Flavia, Artemis micro-vibration environment prediction, spacecraft structures, materials and mechanical testing, Proceedings of a European Conference, Braunschweig, Germany, 4-6 November 1998, Vol. 428, European Space Agency (ESA), Paris, ESA-SP, 1999, pp. 489-495, ISBN: 9290927127.

[2] T. Wacker, L. Weimer, K. Eckert, GOCE platform micro-vibration verification by test and analysis, Proceedings of the European Conference on Spacecraft Structures, Materials and Mechanical Testing, 2005 (ESA SP-581), Noordwijk, Netherlands, 10-12 May 2005.

[3] T. Shimizu, S. Nagata, S. Tsuneta, T. Tarbell, C. Edwards, R. Shine, C. Hoffmann, E. Thomas, S. Sour, R. Rehse, O. Ito, Y. Kashiwagi, M. Tabata, K. Kodeki, M. Nagase, K. Matsuzaki, K. Kobayashi, K. Ichimoto, Y. Suematsu, Image stabilization system for Hinode (Solar-B) solar optical telescope, Solar Physics 249 (2008) 221-232.

[4] N. Jedrich, D. Zimbelman, M. Turczyn, J. Sills, C. Voorhees, B. Clapp, Cryo cooler induced micro-vibration disturbances to the Hubble space telescope, Fifth Cranfield Space Dynamics Conference, July 2002.

[5] C.L. Foster, M.L. Tinker, G.S. Nurre, W.A. Till, Solar-array-induced disturbance of the Hubble space telescope pointing system, Journal of Spacecraft and Rockets 32 (1995) 634-644.

[6] G.S. Aglietti, R.S. Langley, E. Rogers, S.B. Gabriel, Model building and verification for active control of micro-vibrations with probabilistic assessment of the effects of uncertainties, Proceedings of the Institution of Mechanical Engineers, Part C: Journal of Mechanical Engineering Science 218 (2004) 389-399.

[7] A.C.H. Tan, T. Meurers, S.M. Veres, G. Aglietti, E. Rogers, Robust control of micro-vibrations with experimental verification, Proceedings of the Institution of Mechanical Engineers, Part C, Mechanical Engineering Science 219 (5) (2005) 435-460.

[8] L.P. Davis, J.F. Wilson, R.E. Jewell, J.J. Roden, Hubble Space Telescope Reaction Wheel Assembly Vibration Isolation System, NASA Marshall Space Flight Centre, 1986.

[9] S.S. Narayan, P.S. Nair, A. Ghosal, Dynamic interaction of rotating momentum wheels with spacecraft elements, Journal of Sound and Vibration 315 (2008) 970-984, doi:10.1016/j.jsv.2008.02.020.

[10] D. Kamesha, R. Pandiyan, Ashitava Ghosal, Modeling, design and analysis of low frequency platform for attenuating micro-vibration in spacecraft. Journal of Sound and Vibration. 329, (2010) 3431-3450. 10.1016/j.jsv.2010.03.008.

[11] K.C. Liu, P. Maghami, C. Blaurock, Reaction wheel disturbance modeling, jitter analysis, and validation tests for Solar Dynamics Observatory, AIAA Guidance, Navigation and Control Conference and Exhibit, Honolulu, Hawaii, AIAA 2008-7232, 18-21 August 2008, pp. 1-18.

[12] N. Yoshida, O. Takahara, T. Kosugi, K. Ninomiya, T. Hashimoto, K. Minesugi, S. Tsuneta, K. Ichimoto, S. Shimada, Systematic approach to achieve fine pointing requirement of SOLAR-B, IFAC ACA2004 (16th Symposium on Automatic Control in Aerospace), St-Petersburg, Russia, June 14-18, 2004.

[13] S. Miller, P. Kirchman, J. Sudey, Reaction wheel operational impacts on the GOES-N jitter environment, AIAA Guidance, Navigation and Control Conference and Exhibit, Hilton Head, South Carolina, AIAA 2007-6736, 2007, pp. 1-12, 20-23.

[14] O. Takahara, N. Yoshida, K. Minesugi, T. Hashimoto, S. Nagata, M. Koike, S. Shimada, T. Nakaoji, Microvibration transmissibility test of Solar-B, The 24th ISTS (International Symposition on Space Technology and Science), Miyazaki, Japan, May 30-June 6, 2004.

[15] B. Bialke, A compilation of reaction wheel induced spacecraft disturbances, 20th Annual AAS Guidance and Control Conference, AAS Paper 97-038, February 1997.

[16] R.A. Masterson, D.W. Miller, Development and Validation of Empirical and Analytical Reaction Wheel Disturbance Models, Master's Thesis, Department of Mechanical Engineering, Massachusetts Institute of Technology, 1999.

[17] R.A. Masterson, D.W. Miller, R.L. Grogan, Development of empirical and analytical reaction wheel disturbance models, Proceedings of the 40th AIAA/ASME/ASCE/AHS/ASC Structures, Structural Dynamics, and Materials Conference and Exhibit, St. Louis, MO, AIAA 99-1204, April.

[18] R.A. Masterson, D.W Miller, R.L. Grogan, Development and validation of reaction wheel disturbance models: empirical model, Journal of Sound and Vibration 249 (2002) 575-598, doi:10.1006/jsvi.2001.3868.

[19] L.M. Elias, D.W. Miller, A coupled disturbances analysis method using dynamic mass measurement techniques, AIAA/ASME/ASCE/AHS/ASC Structures, Structural Dynamics, and Materials Conference, AIAA2002-1252, 22-25 April 2002.

[20] T. Shigemune, O. Yoshiaki, Experimental and numerical analysis of reaction wheel disturbances, JSME International Journal 46 (2) (2003) 519-526.

[21] H. Youichi, O. Yoshiaki, T. Shigemune, Experiment and analysis of reaction wheel disturbance with periodical torque applied, Transactions of the Japanese Society of Mechanical Engineering 70 (7) (2004) 56-62.

[22] T. Shigemune, K. Masahito, S. Makoto, O. Yoshiaki, Analysis of retainer induced disturbances of reaction wheel, Transactions of the Japanese Society of Mechanical Engineering 71 (1) (2005) 21-28.

[23] G.S. Aglietti, G. Bianchi, J. Barrington-Brown, Impact oscillator based suspension system for a reaction wheel, ECCOMAS Thematic Conference on Computational Methods in Structural Dynamics and Earthquake Engineering, Greece, 22-24 June 2009.

[24] H.C. Corben, Philip Stehle, Classical Mechanics, 2nd Edition, Wiley, New York, 1960, pp. 136-152. 